\section{Evaluation of supporting role of a foldable capsular vitreous body with magnetic resonance imaging in the treatment of severe retinal detachment in human eyes}

${ }^{1}$ State Key Laboratory of Oncology in Southern China, Department of Medical Imaging and Interventional Radiology, Sun Yat-sen University Cancer Center, Sun Yat-sen University, Guangzhou, China

${ }^{2}$ State Key Laboratory of Ophthalmology, Zhongshan Ophthalmic Center, Sun Yat-sen University,

Guangzhou, China

Correspondence: Q Gao, State Key Laboratory of Ophthalmology, Zhongshan Ophthalmic Center, Sun Yatsen University, 54 Xianlie Road, Guangzhou, Guangdong 510060, China Tel: + 86208733 0490; Fax: + 862087333271 .

E-mail: gaoqy@

mail.sysu.edu.cn

Received: 12 April 2010 Accepted in revised form: 11 February 2011 Published online: 18 March 2011

\begin{abstract}
Purpose To determine the supporting role of a novel foldable capsular vitreous body (FCVB) with magnetic resonance imaging (MRI) in the treatment of severe retinal detachment in human eyes.

Methods The study examined nine eyes of nine patients. Among the nine eyes, five had suffered penetrating injuries while four had suffered contusions of the eyeball involving large defects of the retina or choroids. A standard three-port pars plana vitrectomy was performed, FCVB was triple-folded and sent into the vitreous cavity; balanced salt solution (BSS) was injected into the capsule to support the retina. Three cardinal axes of nine eyes were examined using MRI at baseline and at the 3-month follow up.

Results MRI revealed that the signal intensity of the FCVB was similar to the normal vitreous body, with low-signal intensity on T1-weighted image and high-signal intensity on T2-weighted image. In three pre-operative silicone oil- or heavy silicone oil-filled eyes, FCVBs were not fully inflated, and eyeball deformation was observed in one eye. Shifts of three cardinal axes of three eyes (horizontal, anteroposterior, and vertical) according to MRI, were -4.33 , -4.67 , and $-2.67 \mathrm{~mm}$. In the remaining six eyes, FCVBs were well distributed in the vitreous cavity and evenly supported the retina; the cardinal axes of the eyes were similar to pre-operation. Shifts of three cardinal axes of six eyes were $-0.34,-0.34$, and
\end{abstract}

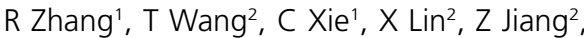
Z Wang ${ }^{2}$, Y Liu², Y Luo ${ }^{2}$, C Long ${ }^{2}$, L He², P Wang ${ }^{2}$ and $\mathrm{Q} \mathrm{GaO}^{2}$
$-0.34 \mathrm{~mm}$. In a total of nine eyes, shifts of three cardinal axes were $-1.67,-1.77$, and $-1.11 \mathrm{~mm}$. Statistically significant difference showed only between the horizontal axis of nine eyes pre-operatively and post-operatively $(P 1=0.041, P 2=0.058, P 3=0.123)$.

Conclusion This study demonstrated the effectiveness of MRI to monitor the supporting role of an FCVB in the treatment of severe retinal detachment in human eyes. Eye (2011) 25, 794-802; doi:10.1038/eye.2011.61; published online 18 March 2011

Keywords: FCVB; human eyes; magnetic resonance imaging

\section{Introduction}

Artificial vitreous substitutes are necessary in the treatment of severe retinal detachment, such as proliferative diabetic retinopathy, proliferative vitreoretinopathy (PVR), and traumatic PVR. ${ }^{1-5}$ Many artificial vitreous substitutes (eg, silicone oil, heavy silicone oil, and hydrogels) are used clinically and in experiments using animals. ${ }^{6-15}$ Among these, silicone oil, introduced by Cibis in 1962, is often injected to an eye suffering from severe retinal or choroidal detachment for an extended time period. Although it has saved many blind patients over the past 50 years, it causes concomitant complications, such as glaucoma, cataracts, corneal degeneration, and emulsification. Moreover, the low-density silicone oil cannot support the inferior retina, 
and it induces a hyperopic shift in the optical system. Although silicone oil is a relatively safe vitreous substitute, it may flow into the anterior chamber, subretina and result in difficult removal because of the free-fluid characteristics within the eye. Therefore, despite a half century of effort to replace the vitreous body of the eye, an ideal and permanent vitreous body has yet to be found. ${ }^{16,17}$

In previous studies, we proposed a new strategy to replace the natural vitreous body using a novel foldable capsular vitreous body (FCVB). ${ }^{18-21}$ The FCVB consisted of a thin, vitreous-shaped capsule with a tube-valve system created using computer and industrial technology. After foldable installation into the eye, a balanced salt solution (BSS) could be injected into the capsule and inflated to support the retina and control the intraocular pressure through the tube-valve system. ${ }^{18}$ In the rabbit model of PVR, we found that the FCVB very closely mimicked the morphology and restored physiological functions, such as support, refraction, and cellular barriers during a 3-month observation period, without the obvious complications commonly induced with traditional silicone oil (see Supplemental Text 1). In addition, the FCVB changes the refraction very little when compared with silicone oil and heavy silicone oil, based on the Gullstrand-Emsley and Liou-Brennan schematic eyes. ${ }^{19}$ Reports from the Chinese State Food and Drug Administration show that the FCVB has good mechanical, optical, and biocompatible properties. ${ }^{20}$ As the FCVB has never been used in eyes worldwide, we conducted an exploratory study of 11 patients implanted by FCVB with BSS in the treatment of severe retinal detachment at Zhongshan Ophthalmic Centre and found it had good flexibility, safety, and efficacy during a 3-month study period. ${ }^{21}$ As the FCVB is made of silicone rubber, magnetic resonance imaging (MRI) might provide valuable information on the role of the FCVB in supporting the retina and eye. ${ }^{22,23}$ Therefore, in this article, we determined the supportive role of intraocular FCVB implantation with MRI in the treatment of severe retinal detachment in human eyes.

\section{Patients and methods}

\section{Study design}

The study protocol was reviewed and approved by the Sun Yat-sen University Medical Ethics Committee (Zhongshan Ophthalmic Centre Medical Ethics (2009) No. 07). The clinical trials strictly adhered to the principles of the World Medical Association's Declaration of Helsinki and have been successfully registered with ClinicalTrials.gov (ClinicalTrials.gov ID: NCT00910702), as well as the Chinese Clinical Trial Register (ChiCTR-TNC-00000396). All patients gave written informed consent.
The severe retinal detachments that were included in this study were not easily re-attached by silicone oil, such as severe posterior scleral ruptures with large retinal absence, severe scleral ruptures with retinal and choroidal detachments, rigid retinal re-detachments (grade D PVR), or inferior holes after silicone oil or heavy oil tamponade. The independent expert committee in Zhongshan Ophthalmic Centre confirmed that the retinal detachments were not easily re-attached by silicone oil. The surgeon ensured this inclusion again during the FCVB surgery. If an eye could be injected with silicone oil, the surgeon could deny the FCVB surgery.

Patients with serious heart, lung, liver, or kidney dysfunction, patients with serious eye inflammation, patients with a single eye, patients with suitable silicone oil-filled eyes, and patients with diseases that the researchers deemed made them unsuitable for participation were excluded from this clinical trial.

\section{Study patients}

Between May 2009 and January 2010, nine patients (nine eyes) were enrolled in this study. The demographic and ocular characteristics of the patients at baseline examination are shown in Table 1. The mean age of the patients was 29.3 years (13-53 years), and all nine (100.0\%) patients were male. Among the nine eyes, five (55.6\%) had incurred penetrating injury, and four $(44.4 \%)$ had incurred contusion of the eyeball combining large defects of the retina or choroids.

\section{Study treatment}

Intervention procedures consisted of vitrectomy, FCVB (Guangzhou Vesber Co. Ltd., Guangzhou, China) implantation, and FCVB removal 3-months later. A standard three-port pars plana vitrectomy was performed, and membrane peeling, retinotomies, and relaxing retinectomies were added if necessary. After scleral incisions of $3 \times 1 \mathrm{~mm}$ were made, the FCVB was triple-folded and sent into the vitreous cavity without air-fluid exchange. About $4.0 \mathrm{ml}$ BSS was injected into the capsule through the valve to push the fluid or subretinal blood to flow out of the eye, and therefore support the retina. The tube and valve were subsequently fixed onto the scleral surface (video 1). Ocular examinations were performed at baseline, day 3, week 1, 2, 4, 6, and 8, and 3 months after FCVB implantation surgery. Visual acuity was graded according to the following system: no light perception was scored as 0 , light perception was scored as 1 , hand motion perception was scored as 2, finger count perception was scored as $3, \geq 0.05$ was scored as 4 , and $\geq 0.1$ was scored as 5 . Before removal of the FCVB at 3 months, the eyes were examined by MRI. 
Table 1 Demographic and ocular characteristics of patients at baseline examination

\begin{tabular}{|c|c|c|c|c|}
\hline Patient & $\begin{array}{c}\text { Age } \\
\text { (Years) }\end{array}$ & Gender & History of surgery & Diagnosis \\
\hline 01 & 53 & Male & $\begin{array}{l}\text { Scleral wound exploration and } \\
\text { repair + eyelid skin laceration suture }\end{array}$ & $\begin{array}{l}\text { Ocular contusion (OD) } \\
\text { 1. scleral and corneal laceration; } 2 \text {. hyphema; } \\
\text { 3. iridodialysis; } 4 \text {. vitreous hemorrhage; } \\
\text { 5. choroidal detachment; } 6 \text {. retinal detachment. }\end{array}$ \\
\hline 02 & 13 & Male & $\begin{array}{l}\text { Corneal wound suturing }+ \text { formation } \\
\text { of anterior chamber; PPV }+\mathrm{C}_{3} \mathrm{~F}_{8}\end{array}$ & $\begin{array}{l}\text { Penetrating ocular injury (OS) } \\
\text { 1. penetrating corneal trauma; } 2 \text {. hyphema; } \\
\text { 3. iridocoloboma; } 4 \text {. aphakic eye; } 5 \text {. vitreous opacity; } \\
\text { 6. retinal detachment. }\end{array}$ \\
\hline 03 & 19 & Male & $\begin{array}{l}\text { Corneal wound suturing; } \\
\text { lensectomy + PPV + silicone oil }\end{array}$ & $\begin{array}{l}\text { Ocular contusion }(\mathrm{OD}) \\
\text { 1. corneal laceration; } 2 \text {. iridocoloboma; } 3 \text {. aphakic eye; } \\
\text { 4. silicone oil eye; } 5 \text {. retinal detachment. }\end{array}$ \\
\hline 04 & 38 & Male & Corneal wound suturing & $\begin{array}{l}\text { Penetrating ocular injury (OD) } \\
\text { 1. penetrating corneal trauma; } 2 \text {. traumatic cataract; } \\
\text { 3. retinal detachment. }\end{array}$ \\
\hline 05 & 13 & Male & $\begin{array}{l}\text { Corneal wound suturing; } \\
\text { PPV + silicone oil }\end{array}$ & $\begin{array}{l}\text { Ocular contusion (OD) } \\
\text { 1. corneal laceration; } 2 \text {. iridocoloboma; } 3 \text {. aphakic eye; } \\
\text { 4. silicone oil eye. }\end{array}$ \\
\hline 06 & 43 & Male & $\begin{array}{l}\text { Scleral wound exploration and } \\
\text { repair + eye skin laceration suture }\end{array}$ & $\begin{array}{l}\text { Ocular contusion (OS) } \\
\text { 1. scleral laceration; } 2 \text {. traumatic cataract; } 3 \text {. vitreous hemorrhage; } \\
\text { 4. choroidal detachment; } 5 \text {. retinal detachment. }\end{array}$ \\
\hline 07 & 25 & Male & $\begin{array}{l}\text { Intraocular foreign body extraction; } \\
\text { PPV; PPV }+\mathrm{C}_{3} \mathrm{~F}_{8} ; \mathrm{PPV}+\text { heavy } \\
\text { silicone oil }\end{array}$ & $\begin{array}{l}\text { Penetrating ocular injury (OS) } \\
\text { 1. penetrating scleral trauma; } 2 \text {. traumatic cataract; } 3 \text {. heavy } \\
\text { silicone oil eye; } 4 \text {. retinal detachment. }\end{array}$ \\
\hline 08 & 19 & Male & $\begin{array}{l}\text { Corneal wound suturing; PPV + } \\
\text { scleral cryosurgery + silicone oil; } \\
\text { silicone oil removal }\end{array}$ & $\begin{array}{l}\text { Penetration ocular injury (OS) } \\
\text { 1. corneal leucoma; } 2 \text {. iridocoloboma; } 3 \text {. aphakic eye; } \\
\text { 4. retinal detachment. }\end{array}$ \\
\hline 09 & 30 & Male & Corneoscleral trauma suturing & $\begin{array}{l}\text { Penetration ocular injury (OS) } \\
\text { 1. penetrating corneoscleral trauma; } 2 \text {. traumatic cataract; } \\
\text { 3. choroidal detachment; } 4 \text {. retinal detachment. }\end{array}$ \\
\hline
\end{tabular}

\section{Magnetic resonance imaging}

The MRI examinations were performed using a 1.5-Tesla unit (Signa, General Electric, CV/i, Waukesha, WI, USA) with a head coil. The imaging protocol in all patients included an axial T2-weighted and an axial T2-weighted fat-suppressed sequence (TR/TE $4100 / 90 \mathrm{~ms}$, echo train length of 15 , field of view $18 \mathrm{~cm}$, slice thickness $3 \mathrm{~mm}$, with no interslice gap, and matrix size $288 \times 224)$, axial, coronal, and sagittal T1-weighted spin-echo (repetition time of $500 \mathrm{~ms}$, echo time of $20 \mathrm{~ms}, 18 \mathrm{~cm}$ field of view, $3 \mathrm{~mm}$ slice thickness with no interslice gap, and a $288 \times 224$ matrix). Following a bolus injection of $0.1 \mathrm{mmol} / \mathrm{kg}$ of either gadolinium dimeglumine (Schering AG, Berlin, Germany) or gadoteric acid (Dotarem; Guerbet, Aulnay, France), higher solution contrast-enhanced T1-weighted spin-echo images using a $288 \times 224$ matrix were acquired in the axial, sagittal, and coronal planes.

\section{Statistics}

Bonferroni multiple comparisons were used to compare pre-operative visual acuity with visual acuity at each time point after FCVB implantation. Wilcoxon SignedRank Test was used for the changes of three cardinal axes of eyes (horizontal, anteroposterior, and vertical) according to MRI. Statistical significance was considered to be present at the $5 \%$ level.

\section{Results}

\section{Follow up examinations}

All the FCVBs were successfully implanted into the eyes and the retinas were reattached during the implantation surgery in all nine eyes. A slight conjunctival hyperaemia appeared by day 7 after surgery in FCVB-filled eyes. No serious complications (eg, corneal keratopathy or intraocular inflammation) were observed. There was no significant 

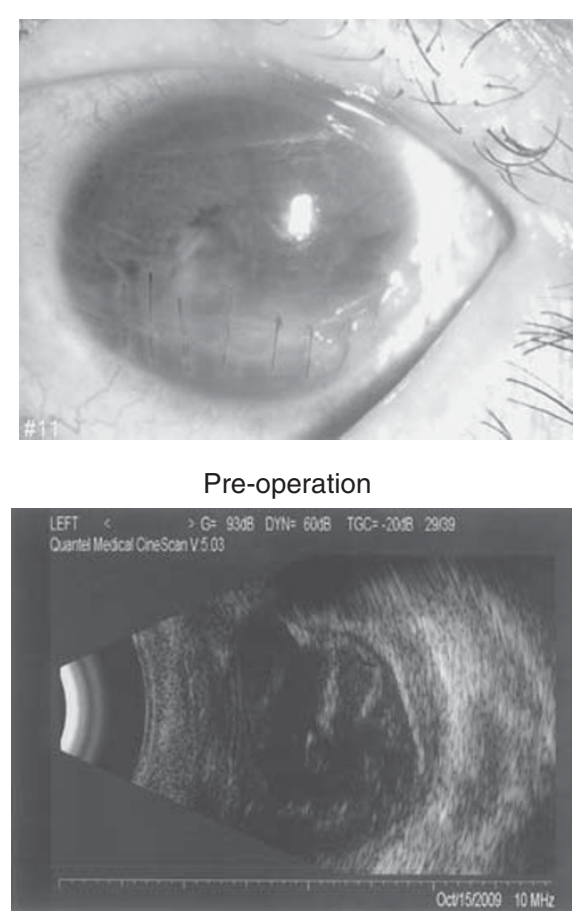

Pre-operation

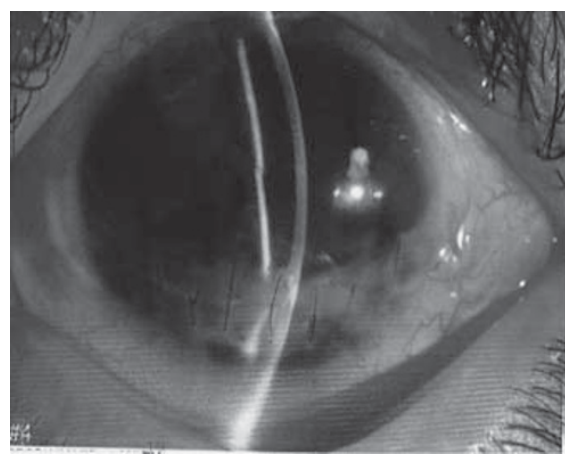

Post-operation at 3 months

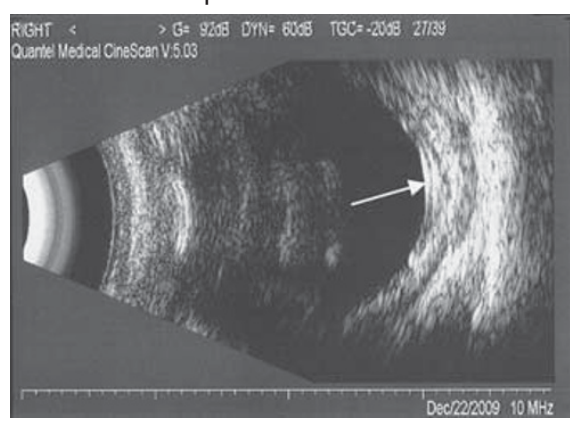

Post-operation at 3 months

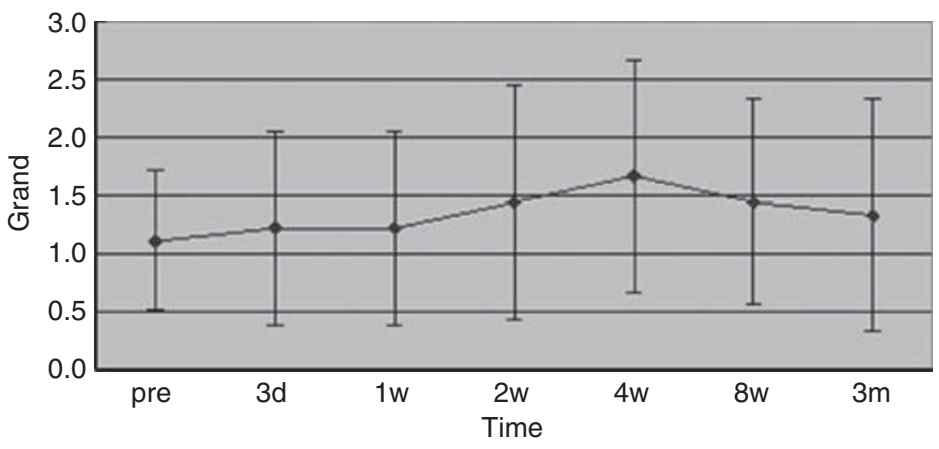

Graded scores of visual acuity during a three-month implantation period

Figure 1 Anterior segment photography, B-scan of case 9 and graded scores of visual acuity during a 3-month implantation period. There was no statistically significant conjunctival congestion, corneal oedema, keratic precipitate, or aqueous flare at 3 months after FCVB implantation. The B-scan showed that a capsule-like arc reflective signal was supporting the retina (arrow). The graded scores of visual acuity at each time point after FCVB implantation do not show a significant difference compared with those at baseline.

conjunctival congestion, corneal oedema, keratic precipitate, or aqueous flare at 3 months after FCVB implantation. No patient showed signs of infection or intolerance to the FCVB material. There was no acute post-operative abnormality by slit lamp biometry observed in any of the cases (Figure 1). The graded scores of visual acuity at each time point did not show a significant difference compared with those at baseline $(P>0.05$, Figure 1$)$. Retinal attachment was found in eight eyes by B-scan at 3 months after implantation. At 3 months after FCVB removal, the 5/9 eyes were still re-attached and 3/9 were re-detached. Among the three re-attached eyes, two eyes received inert gas tamponade after FCVB removal. A good profile of the eyes can be found in the FCVB implantation eye. The B-scan showed that a capsule-like arc reflective signal was supporting the retina (Figure 1). On the basis of the above data, it appears that FCVB can be well distributed in the vitreous cavity and can evenly support the retina. After FCVB removal, eyes are still under observation.

Adverse events were recorded as unbearable foreign body sensations, abnormal bleeding, severe inflammation, endophthalmitis, and sympathetic ophthalmia during clinical trials. No adverse events were observed during the study.

\section{MRI shifts after FCVB implantation}

Because of the high-spatial resolution of MR imaging system, the natural vitreous body clearly appeared in 
vivo. ${ }^{24}$ On MRI in vitro, the FCVB full of BSS appeared as a round object with high-signal intensity on T2-weighted image (Figure 2). The signal intensity of FCVB was similar to that of the natural vitreous body, and three cardinal axes of the FCVB could be measured accurately.

Among the nine eyes, MRI revealed the absence of lens in eight cases, vitreous haemorrhage in one case, subretinal haemorrhage in five cases, retinal detachment in nine cases, and choroidal detachment in two cases.

Two $(22.2 \%)$ eyes had already failed to respond to silicone-oil tamponade. Before FCVB implantation, the two eyes were still filled with silicone oil. In one case,
MRI showed that silicone oil was only filling two-thirds of the vitreal cavity. When the patient reclined, the silicone oil floated above the water, leaving the inferior retina unsupported. The interface of silicone oil and water was clearly observed (Figure 3a). In another case, MRI showed that silicone oil had fully filled the vitreal cavity, while severe retinal detachment was observed. The funnel-shaped shadow in the eye was the detached retina (Figure $4 \mathrm{a}$ ).

One $(11.1 \%)$ eye failed to respond to heavy silicone oil, and MRI showed the detached retina floating in the vitreal cavity. The bright line in the eye is the detached retina (Figure 5a).
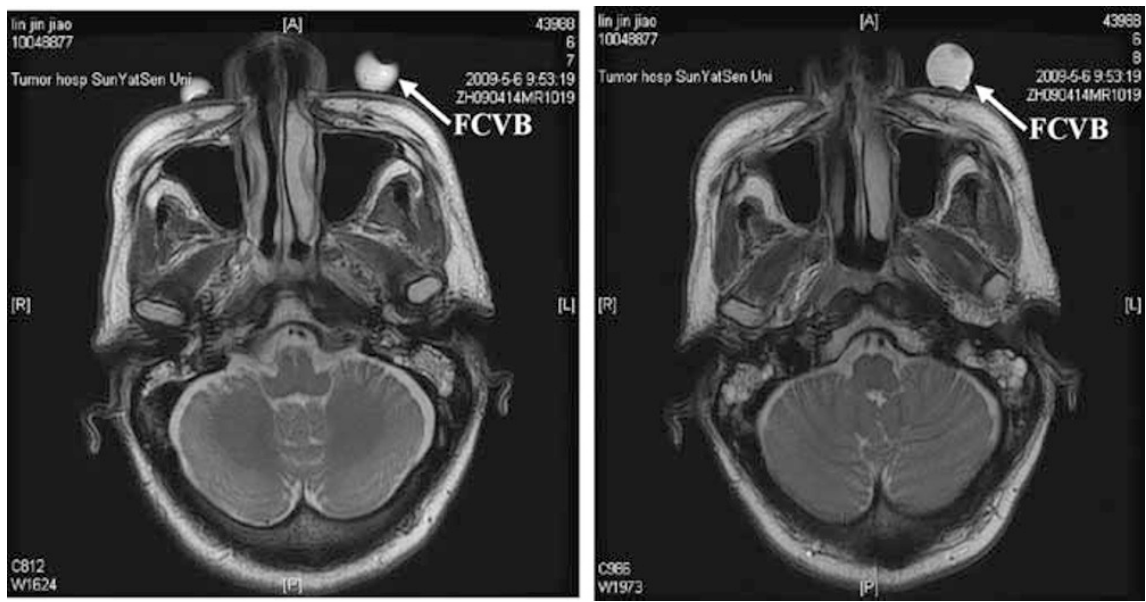

Figure 2 MR images of FCVB in vitro. The FCVB full of BSS appeared as a round object with high-signal intensity on T2-weighted image. The signal intensity of FCVB was similar to that of the normal vitreous body, and three cardinal axes of the FCVB could be measured accurately.
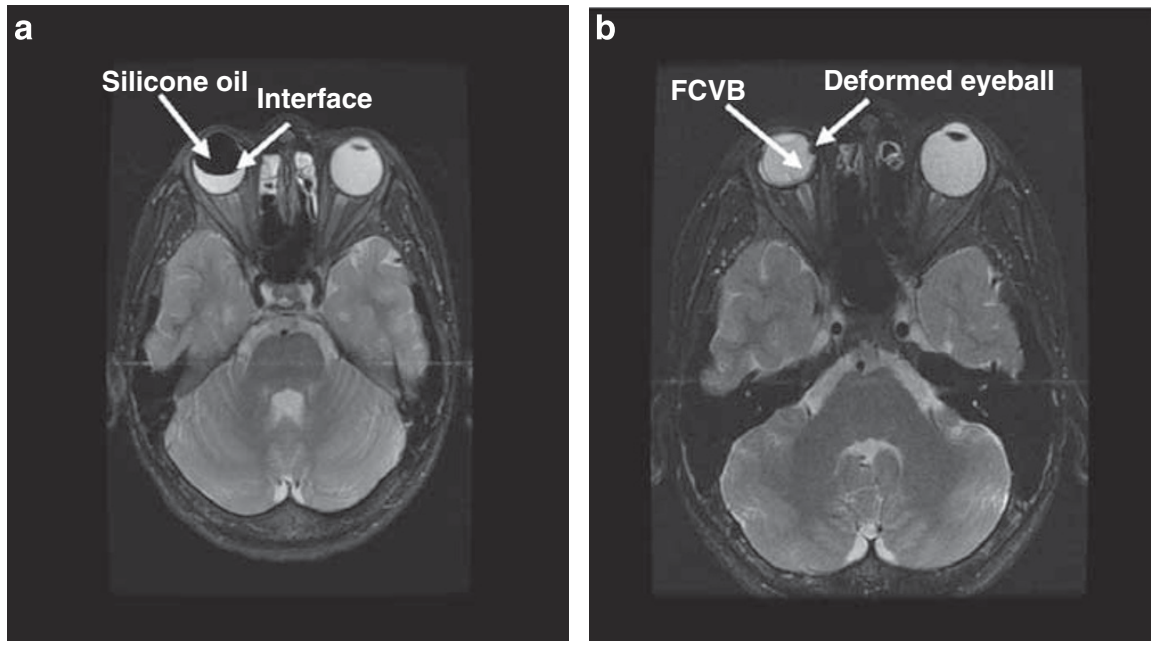

Figure 3 T2-weighted fat-suppressed image of case 5. (a) MRI showed that silicone oil was only filling two-thirds of the vitreal cavity. When the patient reclined, silicone oil floated above the water, leaving the inferior retina unsupported. Interface of silicone oil and water was clearly observed. (b) In this patient, the FCVB was not fully inflated. 

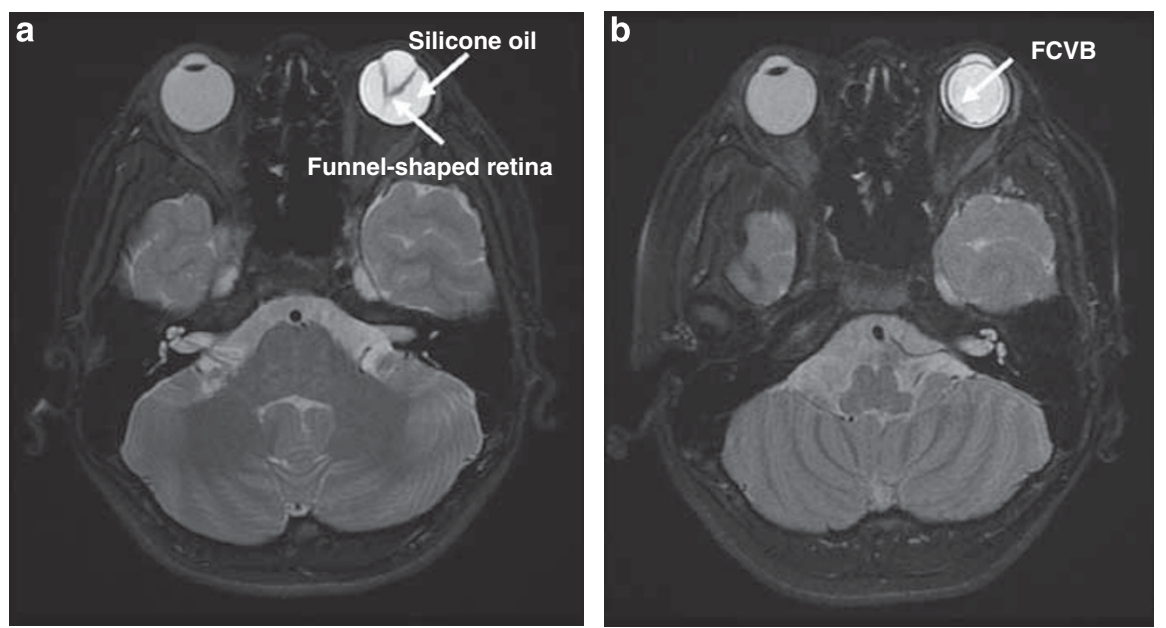

Figure 4 T2-weighted fat-suppressed image of case 3. (a) MRI showed silicone oil fully filled the vitreal cavity, while severe retinal detachment was observed. The funnel-shaped shadow in the eye was the detached retina. (b) An FCVB was fully inflated to support the retina adequately during a 3-month implantation.
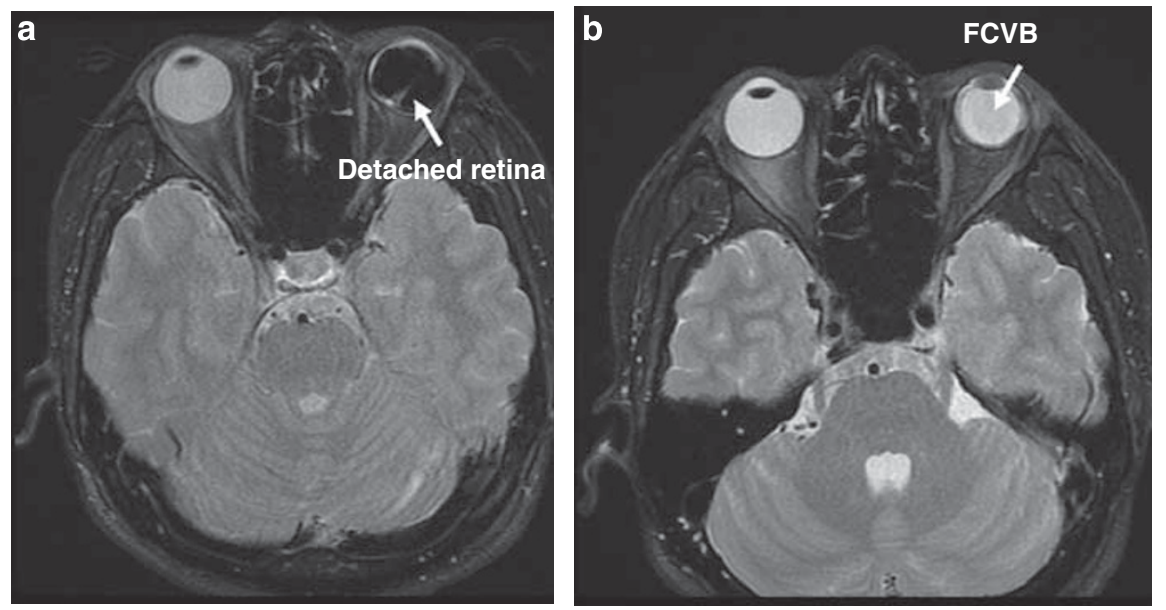

Figure 5 T2-weighted fat-suppressed image of case 7. (a) MRI showed the detached retina floating in the vitreal cavity after heavy oil implantation. The bright line in the eye was the detached retina. (b) An FCVB was fully inflated to support the retina adequately during a 3-month implantation.

MRI was performed on patients with FCVB implantation. The FCVB full of BSS in the eyeball can be observed clearly on MRI. MRI revealed that the FCVB in the eye, which was similar to the normal vitreous body, was of low-signal intensity on T1-weighted images and high-signal intensity on T2-weighted images, without enhancement following contrast medium administration.

In three pre-operative silicone oil- or heavy silicone oil-filled eyes, FCVB were not fully inflated, and eyeball deformation was observed in one eye (Figures $3 b$, $4 b$ and $5 b)$. In the remaining six eyes, FCVBs were well distributed in the vitreous cavity and evenly supported the retina; the cardinal axes of the eyes were similar to pre-operation (Figure 6).
Three cardinal axes of the eyes (horizontal, anteroposterior, and vertical) using MRI are shown in Table 2 and Figure 7. In three pre-operative silicone oil- or heavy silicone oil-filled eyes, the FCVB were not fully inflated, and eyeball deformation was observed in one eye. Shifts of three cardinal axes of these three eyes (horizontal, anteroposterior, and vertical) were -4.33 , -4.67 , and $-2.67 \mathrm{~mm}$, but there was no statistically significant difference between axis oculi of eyes preoperatively and post-operatively $(P 1=0.102, P 2=0.102$, $P 3=0.109$ ). In the remaining six eyes, FCVBs were well distributed in the vitreous cavity and evenly supported the retina; the cardinal axes of the eyes were similar to pre-operation. Shifts of three cardinal axes of six eyes 

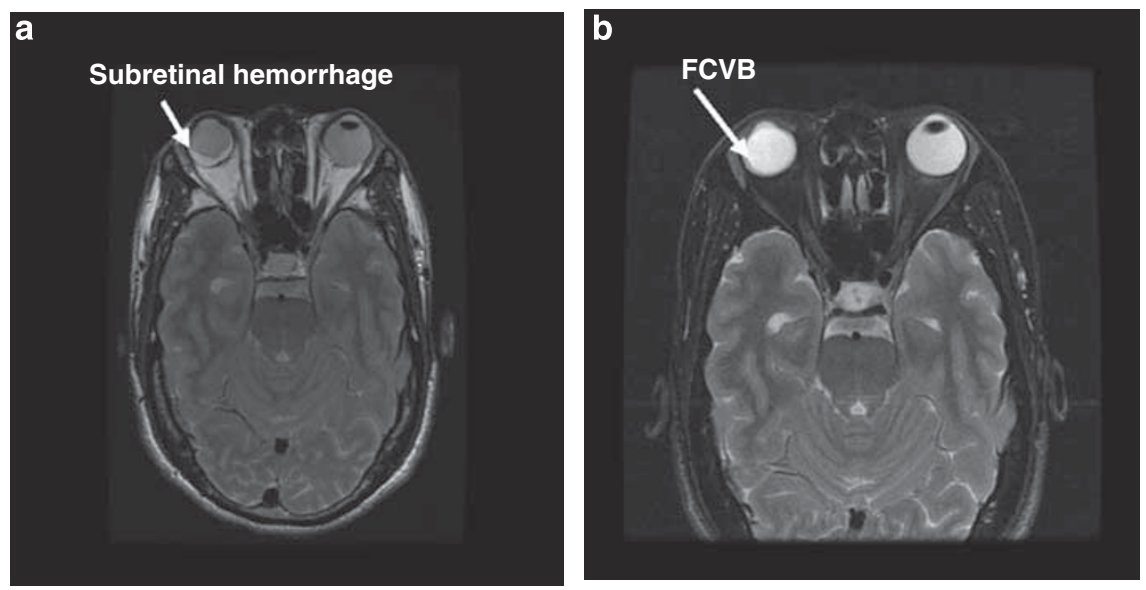

Figure 6 T2-weighted fat-suppressed image of case 4. (a) MRI showed the retina detachment, subretinal haemorrhage, and lack of lens. (b) An FCVB was fully inflated to support the retina adequately during a 3-month implantation.

Table 2 Three cardinal axes of the eyes (antero-posterior, horizontal, and vertical) according to MR imaging

\begin{tabular}{|c|c|c|c|c|}
\hline $\begin{array}{l}\text { Patient } \\
\text { number }\end{array}$ & $\begin{array}{l}\text { Three cardinal } \\
\text { axes of the eyes }\end{array}$ & $\begin{array}{c}\text { Pre- } \\
\text { operation } \\
(\mathrm{mm})\end{array}$ & $\begin{array}{c}\text { Post- } \\
\text { operation } \\
(\mathrm{mm})\end{array}$ & $\begin{array}{c}\text { Contralateral } \\
\text { eye }(\mathrm{mm})\end{array}$ \\
\hline \multirow[t]{3}{*}{01} & Horizontal & 21 & 20 & 22 \\
\hline & Antero-posterior & 20 & 20 & 22 \\
\hline & Vertical & 22 & 20 & 22 \\
\hline \multirow[t]{3}{*}{02} & Horizontal & 22 & 22 & 23 \\
\hline & Antero-posterior & 21 & 20 & 23 \\
\hline & Vertical & 21 & 21 & 23 \\
\hline \multirow[t]{3}{*}{03} & Horizontal & 23 & 19 & 23 \\
\hline & Antero-posterior & 22 & 19 & 23 \\
\hline & Vertical & 22 & 21 & 23 \\
\hline \multirow[t]{3}{*}{04} & Horizontal & 20 & 20 & 21 \\
\hline & Antero-posterior & 19 & 20 & 21 \\
\hline & Vertical & 21 & 20 & 21 \\
\hline \multirow[t]{3}{*}{05} & Horizontal & 24 & 19 & 23 \\
\hline & Antero-posterior & 24 & 16 & 23 \\
\hline & Vertical & 24 & 19 & 23 \\
\hline \multirow[t]{3}{*}{06} & Horizontal & 21 & 20 & 22 \\
\hline & Antero-posterior & 18 & 18 & 22 \\
\hline & Vertical & 17 & 19 & 22 \\
\hline \multirow[t]{3}{*}{07} & Horizontal & 23 & 19 & 23 \\
\hline & Antero-posterior & 22 & 19 & 23 \\
\hline & Vertical & 20 & 18 & 23 \\
\hline \multirow[t]{3}{*}{08} & Horizontal & 20 & 20 & 23 \\
\hline & Antero-posterior & 17 & 17 & 23 \\
\hline & Vertical & 20 & 20 & 23 \\
\hline \multirow[t]{3}{*}{09} & Horizontal & 20 & 20 & 24 \\
\hline & Antero-posterior & 20 & 18 & 22 \\
\hline & Vertical & 20 & 19 & 22 \\
\hline
\end{tabular}

were $-0.34,-0.34$, and $-0.34 \mathrm{~mm}$. There was no statistically significant difference between axis oculi of eyes pre-operatively and post-operatively $(P=0.157$, $P 2=0.414, P 3=0.577)$. Among the nine eyes, shifts of three cardinal axes were $-1.67,-1.77$, and $-1.11 \mathrm{~mm}$. There was statistically significant difference only between horizontal axis of the nine eyes pre-operatively and post-operatively $(P 1=0.041, P 2=0.058, P 3=0.123)$.

\section{Discussion}

In previous studies, we used a new strategy and rationale to fabricate the artificial vitreous substitute using FCVB $^{18-20}$ and first demonstrated that the FCVB can restore the structure and partial functions of a detached retina that are not easily reattached with silicone oil. ${ }^{21}$ Patients in this predicament cannot obtain an intact eyeball and are forced to live with the outcomes of atrophy. This study first showed that FCVBs can have an effective supporting role as determined by MRI in the treatment of severe retinal detachment in human eyes.

More detailed ophthalmic data, such as visual acuity, slit-lamp, fundus, intraocular pressure, OCT can be found in our previous study. ${ }^{21}$

Silicone oil was designed to support the retina by surface tension. As silicone oil is lighter than water, it cannot adequately support the inferior retina as shown in Figure $3 \mathrm{a}$ and $4 \mathrm{a}$. Similarly, although heavier silicone oil is heavier than water, sometimes it failed to support the retina as shown in Figure 5a. Interfaces between water and oil have been found. These two vitreous substitutes may flow into the anterior chamber and subretina, and result in difficult removal because of the free fluid characteristics within the eye.

However, the FCVB was designed to support the retina and the eye adequately by providing a solid arc. The vitreous cavity is full of FCVB. The inferior detached retina was effectively sustained by the FCVB, as shown in Figures 3b, 4b, 5b and 6. Given the high-spatial resolution of MRI, a FCVB full of BSS can be observed clearly in vitro and in vivo. The FCVB full of BSS was revealed as a round object with low-signal intensity on T1-weighted images and high-signal intensity on T2-weighted images. The FCVB capsule can provide the 

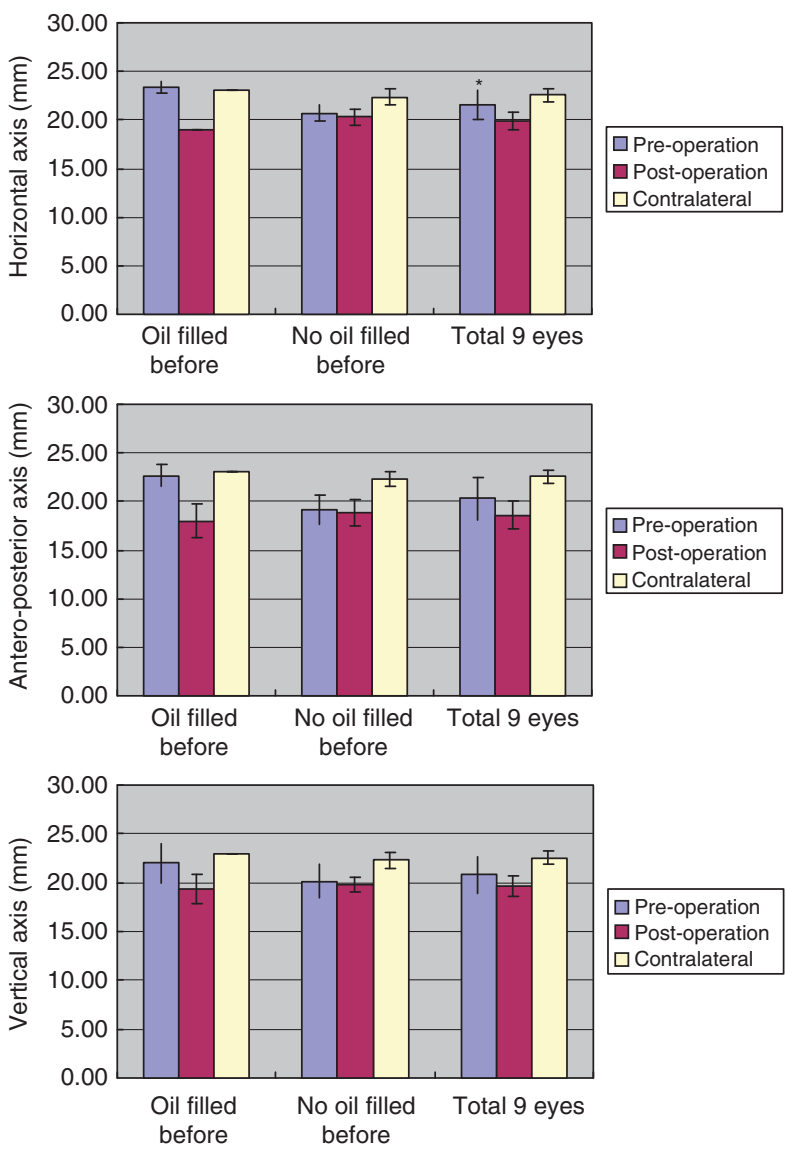

Figure 7 Histogram of three cardinal axes of eyes (horizontal, anteroposterior, and vertical) using MRI. In three pre-operative silicone oil- or heavy silicone oil-filled eyes, shifts of three cardinal axes of three eyes (horizontal, anteroposterior, and vertical) using MRI were $-4.33,-4.67$, and $-2.67 \mathrm{~mm}$. In the remaining six eyes, shifts of three cardinal axes of six eyes were $-0.34,-0.34$, and $-0.34 \mathrm{~mm}$. Among the total nine eyes, shifts of three cardinal axes according to MRI were $-1.67,-1.77$, and $-1.11 \mathrm{~mm}$. There was statistically significant difference only between the horizontal axis of nine eyes pre-operatively and post-operatively $(P 1=0.041, P 2=0.058, P 3=0.123)$.

detached retina with a platform to form a flat scar and a barrier to block cell migration from the retina to the vitreous cavity (see Supplementary Movie 1). In FCVBtreated eyes, because of the constraints of the capsule, it is impossible for the liquid in the FCVB to enter the anterior chamber, while in silicone oil-filled eyes, silicone oil will migrate to the anterior chamber ${ }^{25}(11-49 \%)$ and induce complications, such as glaucoma and corneal keratopathy. Moreover, the FCVB cannot interfere with the anterior chamber or emulsify with time, results that are significantly different from silicone oil. In addition, in our study, patients could maintain a normal position. However, patients with eyes filled with silicone oil must maintain a prone position, which can become very uncomfortable.
In three pre-operative silicone oil- or heavy silicone oil-filled eyes, FCVB were not fully inflated, and eyeball deformation was observed in one eye. In the remaining six eyes, FCVB were well distributed in the vitreous cavity and no eyeball deformation was observed. FCVB may restore the structure better when the eye has not been filled with silicone oil previously.

The FCVB consists of liquid silicone rubber, which is a non-toxic and stable material. ${ }^{20}$ Mechanical properties indicate that liquid silicone rubber has suitable hardness and high-strain capability, which allows the $60 \mu \mathrm{m}$ thin FCVB to stretch its capsule to evenly and gently support the detached retina.

Since $300 \mathrm{~nm}$ small apertures existed in the FCVB capsule, our previous data also showed that the FCVB can sustainably and mechanically release dexamethasone sodium phosphate (DexP) and be used as an intravitreal drug delivery system (DDS) in addition to serving as a vitreous substitute. ${ }^{26}$ As the greatest risk of retinal detachment recurrence occurs 2 months after surgery, the FCVB was designed to be implanted into the eyes for 3 months. As the severity of the current patients was much greater than that of a common patient, future research will focus on the observations of retinal toxicity or mechanical damage and support role of permanent FCVB implantation in the eyes. On the basis of this and a previous study, ${ }^{21}$ a multicenter clinical trial is in progress to ascertain FCVB safety and efficacy as a vitreous substitute in human eyes at 12 hospitals in China.

In conclusion, this study demonstrated that MRI is very useful to monitor the supporting role of an FCVB in the treatment of severe retinal detachment in human eyes.

\section{Summary}

What was known before

- In previous studies, we proposed a new strategy to replace the natural vitreous body using a novel foldable capsular vitreous.

What this study adds

- This study demonstrated the effectiveness of magnetic resonance imaging to monitor the supporting role of an foldable vitreous in the treatment of severe retinal detachment in human eyes.

\section{Conflict of interest}

The authors declare no conflict of interest.

\section{Acknowledgements}

We thank the patients and their families for their continuous support of the study; the medical, examining, 
operating room, anesthesia, and nursing staff at Zhongshan Ophthalmic Center. This study was supported by National High-tech R\&D Program of China (863 Program, 2009AA2Z404).

\section{References}

1 Castellarin A, Grigorian R, Bhagat N, Del Priore L, Zarbin MA. Vitrectomy with silicone oil infusion in severe diabetic retinopathy. Br J Ophthalmol 2003; 87: 318-321.

2 Karel I, Kalvodová B. Long-term results of pars plana vitrectomy and silicone oil for complications of diabetic retinopathy. Eur J Ophthalmol 1994; 4: 52-58.

3 Pastor JC. Proliferative vitreoretinopathy: an overview. Surv Ophthalmol 1998; 43: 3-18.

4 Quiram PA, Gonzales CR, Hu W, Gupta A, Yoshizumi MO, Kreiger AE et al. Outcomes of vitrectomy with inferior retinectomy in patients with recurrent rhegmatogenous retinal detachments and proliferative vitreoretinopathy. Ophthalmology 2006; 113: 2041-2047.

5 Karel I, Michalicková M. Pars plana vitrectomy in the pediatric population: indications and long-term results. Eur J Ophthalmol 1999; 9: 231-237.

6 Cibis PA, Becker B, Okun E, Canaan S. The use of liquid silicone in retinal detachment surgery. Arch Ophthalmol 1962; 68: 590-599.

7 The Silicone Study Group. Vitrectomy with silicone oil or sulfur hexafluoride gas in eyes with severe proliferative vitreoretinopathy: results of a randomized clinical trial. Silicone Study Report 1. Arch Ophthalmol 1992; 110: 770-779.

8 Azen SP, Scott IU, Flynn Jr HW, Lai MY, Topping TM, Benati $\mathrm{L}$ et al. Silicone oil in the repair of complex retinal detachments. A prospective observational multicenter study. Ophthalmology 1998; 105: 1587-1597.

9 Scott IU, Flynn Jr HW, Murray TG, Smiddy WE, Davis JL, Feuer WJ. Outcomes of complex retinal detachment repair using 1000- vs 5000-centistoke silicone oil. Arch Ophthalmol 2005; 123: 473-478.

10 Bhisitkul RB, Gonzalez VH. 'Heavy oil' for intraocular tamponade in retinal detachment surgery. Br J Ophthalmol 2005; 89: 649-650.

11 Mackiewicz J, Mühling B, Hiebl W, Meinert H, Maaijwee K, Kociok $\mathrm{N}$ et al. In vivo retinal tolerance of various heavy silicone oils. Invest Ophthalmol Vis Sci 2007; 48: 1873-1883.

12 Hong Y, Chirila TV, Fitton JH, Ziegelaar BW, Constable IJ. Effect of crosslinked poly (1-vinyl-2-pyrrolidinone) gels on cell growth in static cell cultures. Biomed Mater Eng 1997; 7: 35-47.

13 Hong Y, Chirila TV, Vijayasekaran S, Shen W, Lou X, Dalton PD. Biodegradation in vitro and retention in the rabbit eye of crosslinked poly (1-vinyl-2-pyrrolidinone) hydrogel as a vitreous substitute. J Biomed Mater Res 1998; 39: 650-659.

14 Maruoka S, Matsuura T, Kawasaki K, Okamoto M, Yoshiaki $\mathrm{H}$, Kodama $\mathrm{M}$ et al. Biocompatibility of polyvinylalcohol gel as a vitreous substitute. Curr Eye Res 2006; 31: 599-606.

15 Lam RF, Cheung BT, Yuen CY, Wong D, Lam DS, Lai WW. Retinal redetachment after silicone oil removal in proliferative vitreoretinopathy: a prognostic factor analysis. Am J Ophthalmol 2008; 145: 527-533.

16 Colthurst MJ, Williams RL, Hiscott PS, Hiscott PS, Grierson I. Biomaterials used in the posterior segment of the eye. Biomaterials 2000; 21: 649-665.

17 Soman N, Banerjee R. Artificial vitreous replacements. Biomed Mater Eng 2003; 13: 59-74.

18 Gao Q, Mou S, Ge J, To CH, Hui Y, Liu A et al. A new strategy to replace the natural vitreous by a novel capsular artificial vitreous body with pressure-control valve. Eye 2008; 22: 461-468.

19 Gao Q, Chen X, Ge J, Liu Y, Jiang Z, Lin Z et al. Refractive shifts in four selected artificial vitreous substitutes based on Gullstrand-Emsley and Liou-Brennan schematic eyes. Invest Ophthalmol Vis Sci 2009; 50: 3529-3534.

20 Liu Y, Jiang Z, Gao Q, Ge J, Chen J, Cao X et al. Technical standards of foldable capsular vitreous body regarding mechanical, optical and biocompatible properties. Artif Organs 2010; 34: 836-845.

21 Lin X, Ge J, Gao Q, Wang Z, Long C, He L et al. Evaluation of the flexibility, efficacy, and safety of a foldable capsular vitreous body in the treatment of severe retinal detachment. Invest Ophthalmol Vis Sci 2011; 52(1): 374-381.

22 Singh KD, Logan NS, Gilmartin B. Three-dimensional modeling of the human eye based on magnetic resonance imaging. Invest Ophthalmol Vis Sci 2006; 47: 2272-2279.

23 Patz S, Bert RJ, Frederick E, Freddo TF. T(1) and T(2) measurements of the fine structures of the in vivo and enucleated human eye. J Magn Reson Imaging 2007; 26: 510-518.

24 Cheng HM, Singh OS, Kwong KK, Xiong J, Woods BT, Brady TJ. Shape of the myopic eye as seen with highresolution magnetic resonance imaging. Opt Vis Sci 1992; 69: 698-701.

25 Honavar SG, Goyal M, Majji AB, Sen PK, Naduvilath T, Dandona L. Glaucoma after pars plana vitrectomy and silicone oil injection for complicated retinal detachments. Ophthalmology 1999; 106: 169-176.

26 Gao Q, Liu Y, Ke Q, Wang Z, Xie Z, Jiang Z et al. Dexamethasone sodium phosphate sustained mechanical release in the foldable capsular vitreous body. Invest Ophthalmol Vis Sci 2010; 51: 1636-1642.

Supplementary Information accompanies the paper on Eye website (http://www.nature.com/eye) 